\title{
Unusual Pharyngo-Esophageal Dysphagia Caused by a High-Riding Right Brachiocephalic Artery
}

\author{
Ki Hoon Park, MD¹, Sora Baek, MD, PhD ${ }^{1,2}$, Eun Kyoung Kang, MD, PhD ${ }^{1,2}$ \\ ${ }^{1}$ Department of Rehabilitation Medicine, Kangwon National University Hospital, Chuncheon; \\ ${ }^{2}$ Department of Rehabilitation Medicine, Kangwon National University School of Medicine, Chuncheon, Korea
}

High-riding right brachiocephalic and subclavian arteries are often asymptomatic and rare vascular variations. We present a patient with high-riding right brachiocephalic and subclavian arteries that caused compressive pharyngeal and cervical esophageal dysphagia. An 82-year-old woman presented to our hospital with dysphagia that worsened with solid food. A pulsatile lump was observed on the right neck side. A videofluoroscopic swallowing study showed a deviated food bolus movement anterolaterally from the normal vertical pharyngoesophageal transition, with a filling defect in the lower pharynx through the upper esophagus. Neck computed tomography (CT) showed high-riding right brachiocephalic and subclavian arteries and a tortuous right common carotid artery located adjacent to the trachea in the cervical area. The cervical midline structures were deviated to the left neck side. A neck mass with vascular variation should be considered as a cause of dysphagia that worsens with solid food; CT should be considered to determine its cause.

Keywords Brachiocephalic trunk, Deglutition disorders, Neck mass

\section{INTRODUCTION}

Dysphagia is a commonly encountered disorder with a reported incidence of $12 \%-13 \%$ in a short-term care hospital [1]. Dysphagia can result in serious complications, including malnutrition, dehydration, aspiration, choking, pneumonia, and death. It involves difficulty in the passage of solids or liquids from the mouth to the stomach.
Its common causes include stroke, dementia, Parkinson disease, and compression of swallowing organs.

High-riding right brachiocephalic and subclavian arteries are often asymptomatic and rare vascular variations. Moreover, dysphagia is rarely caused by large vascular variations in the neck area associated with pseudo-aneurysms or a tortuous common carotid artery $[2,3]$. There is no report of high-riding right brachiocephalic and

Received March 25, 2020; Revised June 30, 2020; Accepted July 16, 2020; Published online February 9, 2021

Corresponding author: Sora Baek

Department of Rehabilitation Medicine, Kangwon National University Hospital, Kangwon National University School of Medicine, Baengnyeong-ro 156, Chuncheon 24289, Korea. Tel: +82-33-258-9262, Fax: +82-33-258-9097, E-mail: svpmr@catholic.ac.kr

ORCID: Ki Hoon Park (https://orcid.org/0000-0003-3516-3136); Sora Baek (http://orcid.org/0000-0003-3404-6202); Eun Kyoung Kang (https://orcid. org/0000-0001-5315-1361).

(c) This is an open-access article distributed under the terms of the Creative Commons Attribution Non-Commercial License (http://creativecommons.org/ licenses/by-nc/4.0) which permits unrestricted noncommercial use, distribution, and reproduction in any medium, provided the original work is properly cited. Copyright $\odot 2021$ by Korean Academy of Rehabilitation Medicine 
subclavian arteries causing dysphagia. Here, we present a patient with high-riding right brachiocephalic and subclavian arteries that caused compressive pharyngeal and cervical esophageal dysphagia.

\section{CASE REPORT}

\section{History and physical examinations}

An 82-year-old woman with a history of hypertension, iron deficiency anemia, and chronic bronchitis presented to the Department of Otolaryngology with dysphagia, nonproductive cough, sputum, and hoarseness occurring in the previous month. The dysphagia was accompanied by a feeling of something stuck in the throat. She was on a soft-blend diet since the dysphagia and accompanying feeling worsened with solid food. During the prior month, she had lost weight ( $55 \mathrm{~kg}$ to $42 \mathrm{~kg}$ ). An otolaryngological examination led to a diagnosis of left vocal cord palsy. There was a pulsatile lump on her right neck side. Neck computed tomography (CT) was performed for further evaluation, and she was referred to the Department of Rehabilitation Medicine for dysphagia evaluation. Written informed consent was obtained from the patient.

\section{Videofluoroscopic swallowing study}

During videofluoroscopic swallowing study (VFSS), thin fluids $(2,5$, and $20 \mathrm{~mL})$ and semi-solid food (curdtype yogurt, pudding, soft-blend diet) containing barium were used. VFSS revealed a deviated food bolus movement anterolaterally from the normal vertical pharyngo-
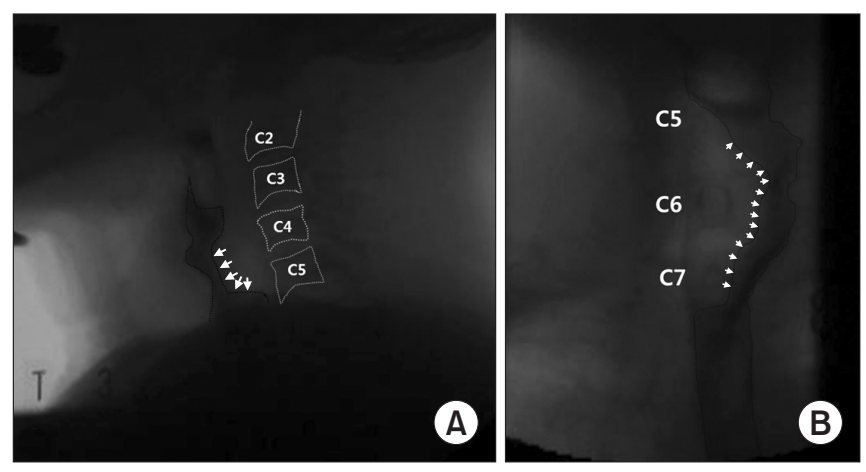

Fig. 1. The esophageal phase of thin fluid swallowing during the videofluoroscopic swallowing study. Both the lateral projection (A) and anterior-posterior views (B) showed a filling defect (white arrow) in the lower pharynx through the upper esophagus at the C4-7 vertebral level. The black dotted line delineates the thin fluid bolus.
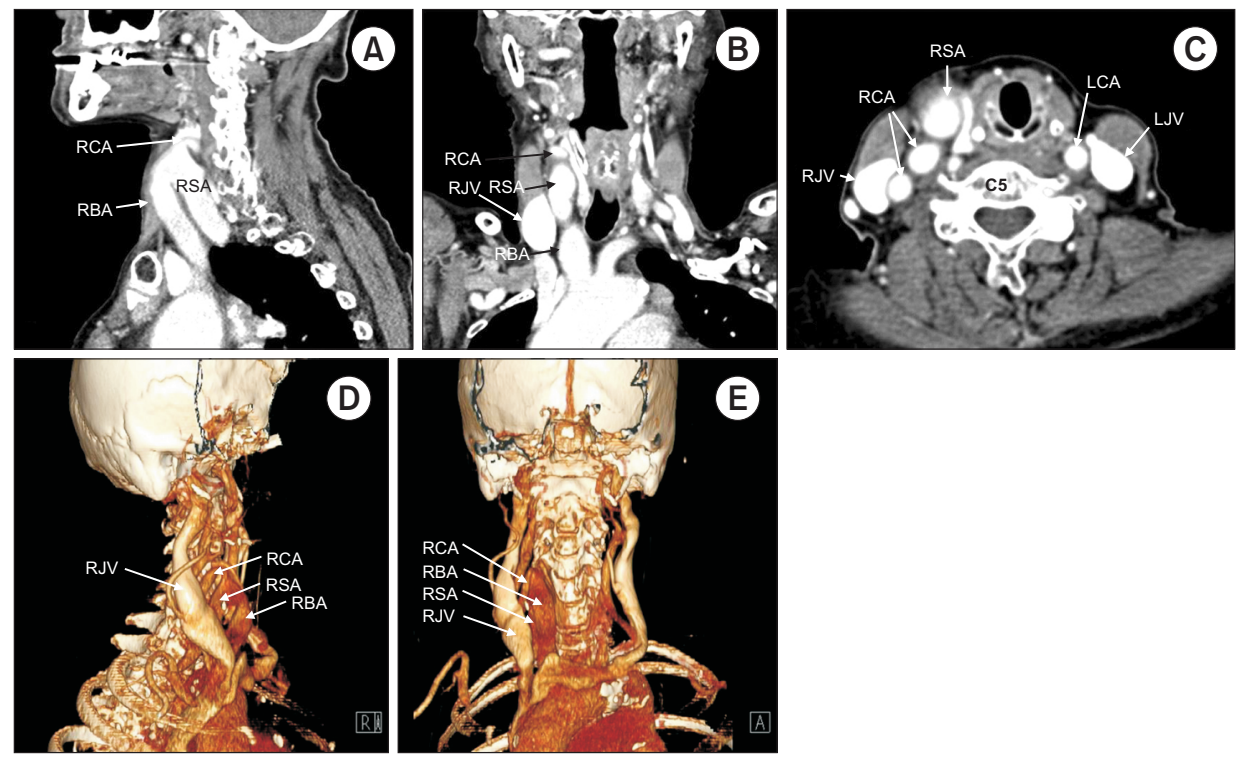

Fig. 2. Enhanced neck computed tomography shows high-riding right brachiocephalic artery (RBA) and right subclavian artery (RSA): (A) sagittal 2D view, (B) coronal 2D view, (C) axial 2D view, (D, E) 3D reconstruction image. (A, D) The RSA branched out from the RBA at the C5 vertebral level and coursed downward posteriorly to the RBA. (B, E) The RSA occupied the right neck area laterally to the trachea. (C) The RSA compressed the midline neck structures, and a left tracheal deviation was observed at the C5 vertebral level. RCA, right common carotid artery; RJV, right jugular vein; LCA, left common carotid artery; LJV, left jugular vein. 
esophageal food transition, with a filling defect in the lower pharynx through the upper esophagus at the C4-7 vertebral level (Fig. 1). These findings were observed with both thin fluid and semi-solid food. Moreover, there was no penetration or aspiration of food materials into the airway or cervical anterior osteophyte in the lateral projection view.

\section{Neck CT}

Neck CT revealed high-riding right brachiocephalic and subclavian arteries and a tortuous common carotid artery (Fig. 2). The right brachiocephalic artery coursed laterally to the trachea up to the $\mathrm{C} 4$ vertebral level where the right subclavian and common carotid arteries branched out. The right subclavian artery coursed downward posteriorly to the brachiocephalic artery. Both the right brachiocephalic and subclavian arteries occupied the right neck area and compressed the midline neck structures with left-side larynx and trachea deviation. At the $\mathrm{C} 7$ vertebral level, the trachea became midline positioned. The esophagus ran along the left tracheal side. Fig. 3 shows the relationship between high-riding large arteries and compressed pharyngo-esophageal structures.

\section{Follow-up}

After performing neck CT and VFSS, we recommended a liquid or semi-solid diet to the patient. With diet modification, her dysphagia symptoms slightly improved, and body weight increased to $44 \mathrm{~kg}$ after 6 months. Despite

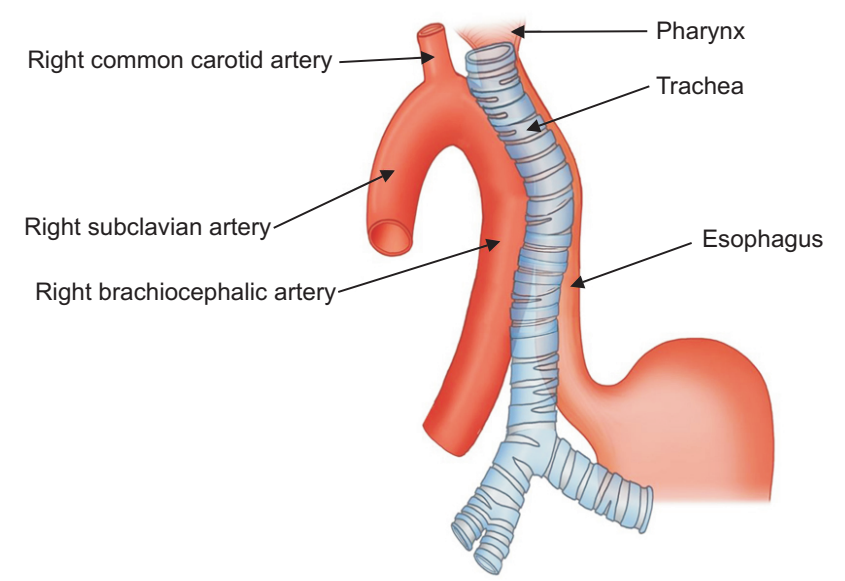

Fig. 3. Tracheal, pharyngeal, and esophageal compression by high-riding right brachiocephalic and subclavian arteries. the slight improvement, overall swallowing difficulty remained. After 1 year, laryngoscopy confirmed that the left vocal cord palsy persisted.

\section{DISCUSSION}

We present a patient with dysphagia caused by compression of the pharynx and cervical esophagus by highriding right brachiocephalic and subclavian arteries.

Dysphagia can be categorized as either oropharyngeal, pharyngeal, or esophageal depending on the location; moreover, it could be neuromuscular or structural depending on the cause. Identifying the dysphagia causes is important for proper management. Surgical management is necessary for dysphagia cases with structural causes, including cervical osteophyte, retropharyngeal abscess, or esophageal cancer [4]. The dietary management of dysphagia caused by structural narrowing consists of a liquid or semi-solid diet. Contrarily, dysphagia involving neuromuscular causes mostly requires a thick fluid diet [5].

Dysphagia caused by extrinsic pharyngeal and esophageal compression can be divided into the intrathoracic and extrathoracic levels. Intrathoracic masses causing compressive dysphagia include mediastinal tumors, aortic aneurysms, dissections, cardiomegaly, and anatomic vascular variants, including an aberrant right subclavian artery [6]. Extrathoracic compressive dysphagia is mostly caused by retropharyngeal structures, including anterior osteophytes of the cervical spine and retropharyngeal abscesses [6]. If these structures are absent, anterolateral neck masses should be examined. Lipoma, hemangioma, thyroid cancer, lymphoma, and lymphadenopathy are often dysphagia-related anterolateral neck masses. Moreover, vascular variations in the anterolateral pharyngeal or esophageal side cause extrathoracic compressive dysphagia. These include pseudo-aneurysms or tortuous common carotid arteries. There has been no report of pharyngo-esophageal compression by vascular variations, such as high-riding right brachiocephalic and subclavian arteries at the extrathoracic level.

High-riding right brachiocephalic and subclavian arteries are rare variations that are often asymptomatic or merely characterized by a pulsatile anterior neck mass. This vascular anomaly has been observed during anterior neck surgery, including tracheostomy, thyroid dis- 
section, or mediastinoscopy, and could cause massive bleeding if it is not identified by the surgeon [7]. There has been no reported case of dysphagia caused by a highriding brachiocephalic artery, which is more pronounced and elongated with aging [8] and might eventually cause compressive dysphagia.

Giant pseudo-aneurysms of the common carotid artery could be associated with rapid progressive dysphagia [2]. In contrast, our case is similar to that of a tortuous common carotid artery. A previous study reported an elderly patient with a long history of dysphagia and compressive pharyngeal and upper esophageal lesions [3]. Neck magnetic resonance imaging showed a tortuous right common carotid artery compressing the esophagus to the left. A barium swallowing test revealed a tight stricture of the lower cervical esophagus with dilatation of the pharynx and upper cervical esophagus proximal to the stricture.

In our case, we observed high-riding right brachiocephalic and subclavian arteries next to the trachea in the cervical area and a deviation of the cervical midline structures to the left neck side. Tracheal deviation in the cervical area is indicative of a large neck mass. The patient presented with dysphagia, and VFSS revealed a filling defect in the pharynx and upper esophagus during swallowing.

Our patient complained of hoarseness, and vocal palsy on the left was confirmed by laryngoscopy, while the abnormally enlarged large arteries were found on the right neck side. The cause of vocal cord palsy is often unidentified, with a tumor or an external mass being one of the common causes. In a previous report, an enlarged esophagus compressed the bilateral recurrent laryngeal nerves that resulted in bilateral vocal cord palsy [9]. In our case, the anomalous arteries in the right neck compressed the midline structure, which might have compressed the left recurrent laryngeal nerve between the adjacent trachea and esophagus, thereby resulting in left vocal cord palsy.

In elderly patients with primary dysphagia that is worsened with solid food who have no history of stroke, dementia, or Parkinson disease, a neck mass should be considered as the underlying cause and CT should be considered.

\section{CONFLICT OF INTEREST}

No potential conflict of interest relevant to this article was reported.

\section{AUTHOR CONTRIBUTION}

Conceptualization: Baek S, Park KH, Kang EK. Writing - original draft: Baek S, Park KH. Writing - review and editing: Baek S, Park KH. Approval of final manuscript: all authors.

\section{REFERENCES}

1. Groher ME, Bukatman R. The prevalence of swallowing disorders in two teaching hospitals. Dysphagia 1986;1:3-6.

2. Gupta V, Niranjan K, Rawat L, Gupta AK. Stent-graft repair of a large cervical internal carotid artery pseudoaneurysm causing dysphagia. Cardiovasc Intervent Radiol 2009;32:558-62.

3. Gupta A, Winslet MC. Tortuous common carotid artery as a cause of dysphagia. J R Soc Med 2005;98:2756.

4. Gamache FW Jr, Voorhies RM. Hypertrophic cervical osteophytes causing dysphagia: a review. J Neurosurg 1980;53:338-44.

5. Bakheit AM. Management of neurogenic dysphagia. Postgrad Med J 2001;77:694-9.

6. Carucci LR, Turner MA. Dysphagia revisited: common and unusual causes. Radiographics 2015;35:105-22.

7. Upadhyaya PK, Bertellotti R, Laeeq A, Sugimoto J. Beware of the aberrant innominate artery. Ann Thorac Surg 2008;85:653-4.

8. Dua SG, Purandare NC, Pramesh CS. Incidental detection of high-riding innominate artery and bilateral retropharyngeal carotid arteries: radiological findings and clinical relevance. Clin Radiol 2011;66:685-7.

9. Chegar BE, Emko P. Bilateral vocal cord paralysis secondary to esophageal compression. Am J Otolaryngol 2004;25:361-3. 